\title{
MARICULTURA EM SANTA CATARINA: A CADEIA PRODUTIVA GERADA PELO ESFORÇO COORDENADO DE PESQUISA, EXTENSÃO E DESENVOLVIMENTO TECNÓLOGICO
}

\author{
Gilberto José Pereira Onofre de Andrade \\ Universidade Federal de Santa Catarina \\ gilberto.andrade@ufsc.br
}

Resumo

Atualmente a cadeia de produção de moluscos em Santa Catarina é responsável por $98 \%$ de toda a produção nacional. Este é um excelente exemplo de como um trabalho organizado e coordenado entre instituições de pesquisa e extensão podem efetivamente solucionar problemas reais e criar novas possibilidades de trabalho e geração de empregos. Dentre as instituições que, nas últimas 3 décadas, têm se dedicado ao desenvolvimento tecnológico voltado para as necessidades desta cadeia de produção, pode-se destacar o papel da UFSC, como um elo fundamental para criação e manutenção desta atividade, que, através do Laboratório de Moluscos Marinhos, tem se concentrado na pesquisa e extensão relacionada a produção de moluscos bivalves. Neste trabalho se apresenta o histórico de desenvolvimento da cadeia produtiva, assim como, se traz luz para as possibilidades de crescimento futuro desta importante atividade econômica no estado.

Palavras-chave: Moluscos Bivalves. Sementes de Moluscos. Malacocultura. Maricultura. Aquicultura.

\section{MARICULTURE IN SANTA CATARINA: THE PRODUCTIVE CHAIN GENERATED BY THE COORDINATED EFFORT OF RESEARCH, EXTENSION AND TECHNOLOGICAL DEVELOPMENT}

\begin{abstract}
Currently, the mollusc production chain in Santa Catarina accounts for $98 \%$ of all national production. This is an excellent example of how an organized and coordinated work between research and extension institutions can effectively solve real problems and bring up new possibilities for work and job creation. Among the institutions that have been dedicated, in the last three decades, to technological development focused on the needs of this production chain, we can highlight the role of UFSC, as a fundamental link for the creation and maintenance of this activity, through the Marine Molluscs Laboratory, has concentrated on the research and extension related to the production of bivalve molluscs. This paper presents the entire history of the development of the productive chain, as well as the possibilities of future growth of this important economic activity in the state.

Keywords: Bivalve Molluscs. Molluscs Spats. Shellfish Production. Mariculture. Aquaculture.

\section{MALACOCULTURA EN SANTA CATARINA: LA CADENA PRODUCTIVA GENERADA POR EL ESFUERZO COORDENADO DE INVESTIGACIÓN, EXTENSIÓN Y DESARROLLO TECNOLÓGICO}

\section{Resumen}

Actualmente la cadena de producción de moluscos en Santa Catarina es responsable por 98\% de toda la producción nacional. Este es un excelente ejemplo de cómo un trabajo organizado y coordinado entre instituciones de investigación y extensión pueden solucionar efectivamente problemas reales y criar nuevas posibilidades de trabajo y creación de empleos. Entre las instituciones que, en las ultimas décadas, se han dedicado al desarrollo tecnológico frente a las necesidades de esta cadena de producción, es posible destacar el papel de la UFSC, como un enlace fundamental para la creación y mantención de esta actividad, que, a través del Laboratorio de Moluscos Marinos, se ha concentrado en investigación y extensión relacionada con la producción de moluscos bivalvos. En este trabajo se presenta un histórico de desarrollo de la cadena productiva, de este modo, se trae luz para las posibilidades de crecimiento futuro de esta importante actividad económica en el estado.

Palavras clave: Moluscos Bivalvos. Semillas de Moluscos. Malacocultura. Maricultura. Acuicultura. 
Maricultura em Santa Catarina: a cadeia produtiva gerada pelo esforço coordenado de pesquisa, extensão e desenvolvimento tecnólogico

\section{INTRODUÇÃO}

Assim como os agricultores cultivam a terra com sementes das mais diversas plantas visando uma farta colheita de alimentos, em Santa Catarina, no Brasil também se plantam sementes no mar!

Devido à super explotação dos oceanos, pela atividade da pesca extrativa, os volumes de "produção" estão em dramático declínio em todo o mundo. Assim, sob as necessidades atuais de incremento na produção de alimentos, o mar não pode mais ser visto apenas como um local de coleta, mas sim como um campo fértil para o plantio, um oceano de possibilidades. A aquicultura representa uma das últimas alternativas, ainda não saturadas, quanto ao incremento da produção de alimento de alta qualidade nutricional.

As sementes plantadas no mar de Santa Catarina, no Brasil, são destinadas à atividade aquícola de malacocultura, nome específico dado à atividade de cultivo de moluscos. Esta é uma forma de Maricultura onde não há entrada de alimento ou fertilizantes, sendo este um dos principais custos de outros cultivos. Nesta atividade, dentre os produtos de interesse comercial, destacam-se os moluscos bivalves: mexilhões (Perna perna), a ostra do pacífico (Crassostrea gigas), a ostra nativa (Crassostrea gasar e C. rhizophorae) e as vieiras (Nodipecten nodosus).

Os moluscos bivalves são animais curiosos, pode-se dizer que cada animal é uma pequena unidade filtradora de água. Possuem um sistema para captar a água e fazê-la passar por suas brânquias, que funcionam como um filtro, sendo responsáveis pela respiração, e retenção de partículas em suspensão: o fitoplâncton, detritos orgânicos e inorgânicos (HUNER \& BROWN, 1985). As partículas retidas são digeridas, uma parte é absorvida e outra eliminada em forma de fezes. O material não ingerido é eliminado como pseudo-fezes. Ambas são reaproveitadas como insumo por: plantas aquáticas, crustáceos, micro-organismos e outros animais.

Dependendo da espécie e idade, um único animal adulto pode filtrar, por hora, até 20 litros de água (QUAYLE \& NEWKIRK, 1989). Considerando-se que uma fazenda padrão, com 1-2 hectares de lâmina de água, possa ter algo em torno de 40.000 bivalves, pode-se dizer que estes se constituem como um verdadeiro exército da limpeza, podendo filtrar até 800 mil litros de água por hora.

Por fim, a atividade também é tida como uma forma de "biosequestro" de carbono, incorporando-o em suas conchas por longo período (WOLF \& BEAUMONT, 2011). Segundo Hinkel(2008) para cada $100 \mathrm{~g}$ de concha de ostras do pacífico, tem-se $12 \mathrm{~g}$ de carbono absorvido de forma permanente. Assim, proporcionando mais um impacto positivo e caracterizando-se como um tipo de cultivo benéfico ao meio ambiente. 
Maricultura em Santa Catarina: a cadeia produtiva gerada pelo esforço coordenado de pesquisa, extensão e desenvolvimento tecnólogico

Outro aspecto surpreendente quanto aos bivalves, diz respeito à estratégia reprodutiva e sua espetacular capacidade de geração de descendentes. $O$ destaque se dá justamente na quantidade de gametas, produzidos pelos animais já maduros para reprodução.

Em ambiente natural, no fim do estágio larval, os indivíduos se fixam definitivamente em algum tipo de substrato, normalmente nas rochas de costão. Portanto, na época de reprodução, os indivíduos não podem se locomover e buscar sua parceria para reproduzir. Em contraponto, visando aumentar as chances para que, de fato, ocorra a reprodução a natureza garantiu-lhes uma capacidade incomum para produção de gametas. Assim, a capacidade de geração de descendentes é realmente espetacular. Por exemplo, um único casal de ostras do pacífico pode gerar 50 milhões de larvas, ou até o dobro. (HELM \& BOURNE, 2004). Entretanto, por estarem soltos na coluna da água, estes potenciais futuros descendentes estão à mercê da sorte. Ao final, da quantidade total de larvas produzidas, apenas uma parcela mínima consegue se desenvolver até apresentar o formato de uma ostra.

Considerando todos os benefícios envolvidos em um cultivo bivalves, onde: o alimento é farto e gratuito no mar, não há necessidade de uso de remédios, causa impactos positivos no meio-ambiente e ainda por cima os animais tem uma incrível capacidade de reprodução; sob a perspectiva de formação da base para que se estabeleça uma cadeia produtiva, eis que surgiu uma pergunta intrigante: Como seria a produtividade na reprodução sob condições controladas, com baixo risco de predadores ou outros agentes que ofereçam perigo ao processo de fecundação?

Este questionamento surgiu muito tempo atrás, trouxe motivação para as discussões e fez parte da base orientadora para o desenvolvimento de objetivos e trabalhos conjuntos, coordenados entre diferentes instituições de pesquisa e extensão. Dentre todos que participaram, cabem destacar o papel vital da Universidade Federal de Santa Catarina-UFSC e Empresa de Pesquisa Agropecuária e Extensão Rural de Santa Catarina-EPAGRI, que somaram esforços múltiplos ao longo dos últimos 30 anos, visando contribuir para o desenvolvimento e fortalecimento da maricultura no estado. Cada parte contribuiu da melhor forma possível no sentido de pesquisar, desenvolver e disseminar as bases tecnológicas para viabilizar a criação de uma nova atividade econômica no estado.

Haja vista a importância e complexidade para o desenvolvimento de uma nova atividade, muitos foram os aspectos estruturais discutidos, definidos, testados e validados. A obtenção de formas jovens foi uma questão especialmente crucial, pois deveria permitir um fornecimento sistemático e contínuo, que trouxesse segurança tanto para a cadeia produtiva, como tornasse a atividade atrativa. Nesse sentido, coube a UFSC a tarefa de pesquisa e desenvolvimento do 
Maricultura em Santa Catarina: a cadeia produtiva gerada pelo esforço coordenado de pesquisa, extensão e desenvolvimento tecnólogico

pacote tecnológico de toda a produção, com foco particular no estabelecimento do sistema produtivo deste insumo básico para viabilizar a cadeia, nesse caso, as sementes de ostras, mexilhões e vieiras.

Já se passaram mais de 3 décadas desde que o processo teve inicio. Desde então foram muitas tentativas, erros, desistências, persistências e acertos até chegar-se a situação atual: hoje o estado de Santa Catarina é o líder nacional na produção de moluscos bivalves e o segundo maior produtor da América Latina, FAO (2016). Com base em dados da EPAGRI (2016), a produção de moluscos bivalves (ostras, mexilhões e vieiras) em Santa Catarina, no ano de 2015, gerou uma movimentação financeira estimada em $\mathrm{R} \$ 78.895 .697,64$ com envolvimento direto de 2.315 pessoas e a produção de um volume de 20.438 toneladas de moluscos bivalves.

Portanto, a criação e o desenvolvimento desta atividade tiveram forte impacto econômico e socioambiental e, ocorreu como resultado do grande esforço coordenado das instituições de pesquisa e extensão envolvidas, onde, notoriamente a UFSC assumiu um papel determinante para a viabilização do cenário atual. Para que se possa entender como tudo isso ocorreu, no próximo tópico é apresentada uma retrospectiva dos fatos mais importantes.

\section{SURGIMENTO E EVOLUÇÃO DA CADEIA DE PRODUÇÃO DA MALACOCULTURA EM SANTA CATARINA-BRASIL}

As primeiras experiências mais consistentes de cultivo de bivalves no Brasil se deram no inicio da década de 70, com a tentativa de se cultivar a ostra do mangue, C. rhizophorae, nos estados da Bahia, São Paulo e Santa Catarina (POLI, 2004), as quais, infelizmente, não obtiveram grande êxito. Seguindo com as tentativas, ao longo das décadas de 70 e 80, a UFSC em parceria com a ACARPESC (atual EPAGRI) deram sequência aos trabalhos com as ostras nativas em Santa Catarina. Todavia, os resultados alcançados ainda foram pouco motivadores, devido às dificuldades presenciadas naquele momento.

Em paralelo a isso, nesta fase inicial de prospecção das possibilidades voltadas para criação de bivalves, em 1974, houve a primeira importação e introdução da espécie C. gigas no Brasil, pelo Instituto de Pesquisas da Marinha, no litoral de Cabo Frio RJ. Já no ano seguinte, 1975, iniciaram as pesquisas na região de Cananéia, com indivíduos importados do Japão, pelo Instituto de Pesca de São Paulo. Esta espécie é campeã de produtividade, além da grande 
Maricultura em Santa Catarina: a cadeia produtiva gerada pelo esforço coordenado de pesquisa, extensão e desenvolvimento tecnólogico

capacidade de adaptação ambiental também tem um rendimento de carne superior às demais espécies de ostra.

Durante a década de 80 , as atenções naturalmente se voltaram para esta espécie, a ostra do pacífico, com a grande possibilidade de sucesso na implementação da atividade de ostreicultura no Brasil. Diretamente alinhada a esses objetivos a UFSC, através do Departamento de Aquicultura, mais especificamente do Laboratório de Cultivo de Moluscos Marinhos (LCMM), sem perder o foco nas espécies nativas, passou também a direcionar esforços para a pesquisa e desenvolvimento tecnológico no sentido de viabilizar a produção desta espécie no estado. Considerando que se trata de uma espécie exótica, diferentemente das espécies nativas, não era possível obter sementes diretamente no ambiente. Portanto, eis que se definiu um grande desafio para o cultivo de ostras do pacífico no Brasil: conseguir formas jovens, em bases regulares, que viabilizassem a produção em escala comercial.

Diante desse desafio o LCMM, em paralelo às pesquisas com as nativas, passou a se concentrar em desenvolver um pacote tecnológico para viabilizar a produção de formas jovens desta, e das demais espécies de moluscos. Em 1985 surgiu o "Projeto Ostra" voltado para o cultivo de ostra do mangue de forma consorciada com camarão. Em 1988 foi criado o $1^{\circ}$ condomínio de Maricultura do Brasil, o Condomínio de Pesca e Maricultura Baía Norte, o qual estabeleceu uma relação importante entre os pescadores da região e os pesquisadores da UFSC.

Figura 1: Evolução da produção de moluscos bivalves produzidos em Santa Catarina.

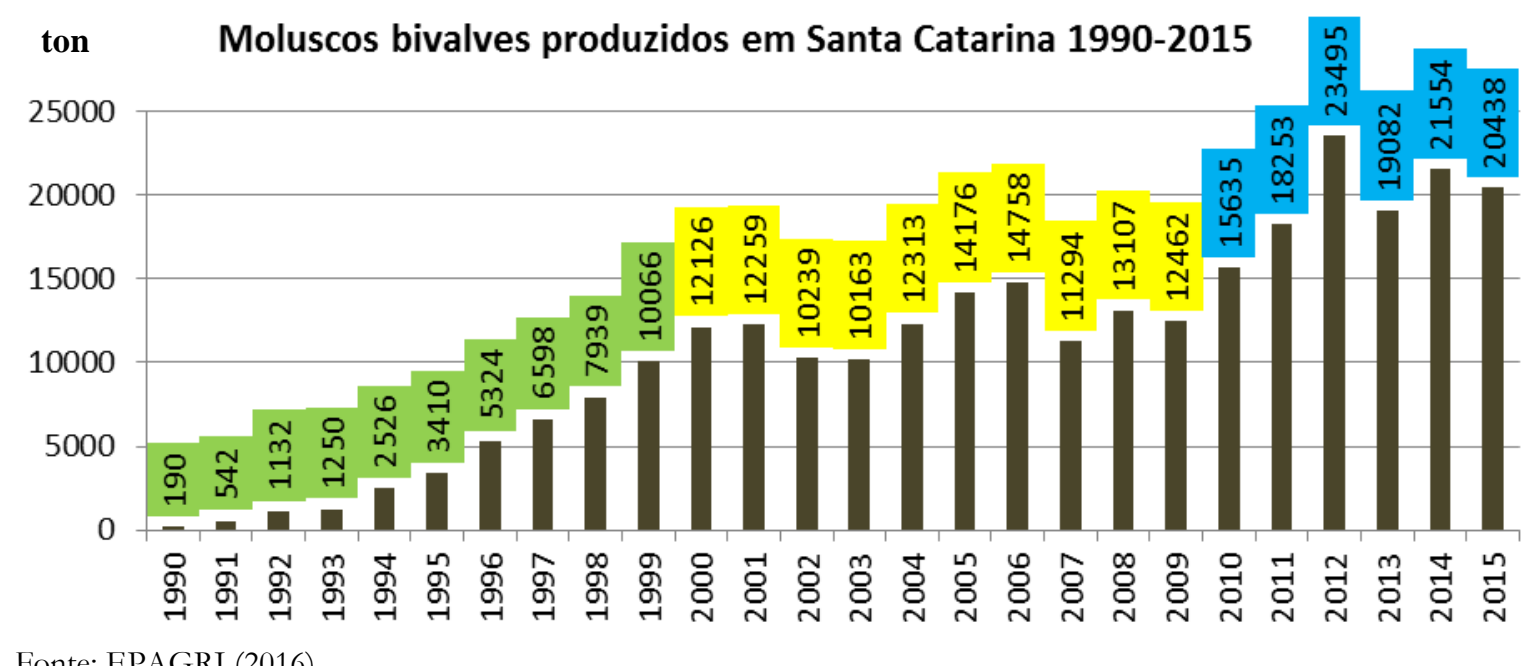

Fonte: EPAGRI (2016)

O início da década de 1990 representa o começo da atividade de maricultura em escala comercial, tanto da produção de ostras como de mexilhões e vieiras. Na Figura 1, fica nítido que 
Maricultura em Santa Catarina: a cadeia produtiva gerada pelo esforço coordenado de pesquisa, extensão e desenvolvimento tecnólogico

esta década ficou fortemente marcada justamente por um veloz crescimento dos volumes de produção, quando então a produção cresceu de 190 toneladas, no ano de 1990, para um total de 10.066 toneladas, no ano de 1999. Trata-se de um incremento da ordem de 5200\% durante uma única década, o que nos dá uma taxa média de crescimento de 520\% ao ano. Ainda nesta década houve um fato que merece ser comentado, que foi a participação da UFSC no STTP (Sellfish Tecnology Transfer Program) e subsequentemente no BMLP (Brazilian Mariculture Linkage Program) desenvolvido em parceria com a CIDA (Canadian Internacional Development Agency). Este apoio técnico e financeiro dos canadenses permitiu ao LCMM, no ano de 1995, a estruturação física de um laboratório pleno para a produção em escala de formas jovens de bivalves. Esta parceria não foi apenas importante no quesito infraestrutura tecnológica, mas também no aspecto de capacitação de mão de obra especializada. Ao final deste período, já se podia dizer que havia uma nova atividade, de forte impacto, tanto econômico quanto sócioambiental, em pleno desenvolvimento no estado de Santa Catarina.

$\mathrm{Na}$ década de 2000 o mercado parou de crescer e a demanda ficou estabilizada entre $11 \mathrm{e}$ 14 mil toneladas conforme Figura 1. Foi um período de consolidação e aprimoramento dos processos produtivos, porém sem grandes perspectivas de crescimento no mercado interno.

Por fim, a década atual tem sido marcada por um processo de qualificação de toda a cadeia produtiva e um leve aumento da demanda. Cabem destacar, o PLDM (Plano Local de Desenvolvimento da Maricultura) que contribuiu para regularização de toda a atividade, assim como, o Programa Nacional de Controle Higiênico Sanitário de Moluscos Bivalves (PNCMB), que busca a adequação da cadeia às exigências vigentes em outros mercados externos como os países da União Europeia (SOUZA et al., 2014). Mais recentemente, em 2016, foi criada a Lei 16.874, que declara a maricultura como atividade de interesse social e econômico e estabelece as condições para seu desenvolvimento sustentável em Santa Catarina.

A Figura 2 apresenta uma retrospectiva da cadeia produtiva de moluscos em Santa Catarina. Em linhas gerais, a década de 1970 foi importante para prospecção das possibilidades e busca de informações, foi o período da Investigação. A década seguinte, de 1980, foi justamente o período para se aprofundar as investigações e identificar, de fato, quais eram as oportunidades reais e factíveis, foi o período do Desenvolvimento. Já a década de 1990 ficou marcada pelo crescimento vertiginoso da produção, foi o período do Crescimento. Na década de 2000 podese dizer que houve uma estabilização da demanda e consolidação dos processos produtivos, foi o período do Aprimoramento. Já na década de 2010, o foco tem sido na organização e regularização da cadeia produtiva como um todo. O objetivo maior tem sido o planejamento e a 
Maricultura em Santa Catarina: a cadeia produtiva gerada pelo esforço coordenado de pesquisa, extensão e desenvolvimento tecnólogico

ordenação de toda atividade, visando ganhar confiabilidade e maior competitividade a fim de que se possa qualificar para o mercado externo. Este tem sido o período da Qualificação.

Figura 2: Retrospectiva do surgimento e evolução da cadeia de produção da malacocultura em SC.

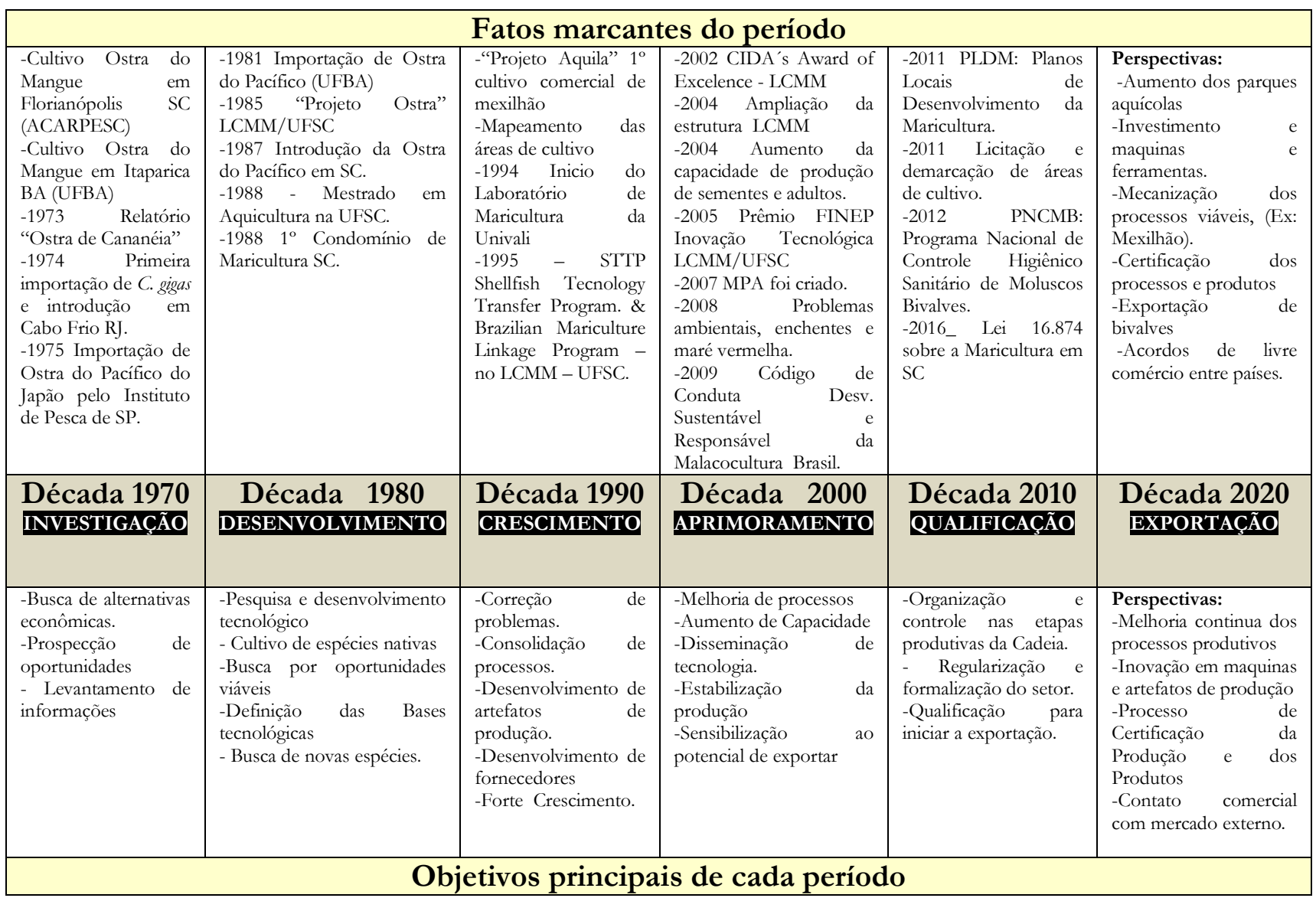

No desenvolvimento da Cadeia de Produção de Moluscos, o sucesso do trabalho coordenado, de pesquisa e extensão, das instituições envolvidas outorgou ao estado de Santa Catarina a posição de líder isolado deste tipo de mercado no Brasil, com cerca de $98 \%$ de toda a produção nacional (IBGE 2015). Florianópolis passou a ser reconhecida como a "Capital da Ostra", sendo que a principal festa da cidade - Festa Nacional da Ostra e Cultura Açoriana - a FENAOSTRA já se encontra em sua $17^{a}$ edição, em 2016. Um evento que atrai milhares de participantes a cada ano e multiplica o reconhecimento de Florianópolis, como um centro de excelência de produção de bivalves, por todo o Brasil e até no exterior. 
Maricultura em Santa Catarina: a cadeia produtiva gerada pelo esforço coordenado de pesquisa, extensão e desenvolvimento tecnólogico

\section{DIAGNÓSTICO DO POTENCIAL DE CRESCIMENTO DA CADEIA DE PRODUÇÃO DE MOLUSCOS EM SANTA CATARINA}

O Brasil já é um protagonista na produção e exportação de diferentes tipos de proteína animal, como é o caso da carne bovina, com o segundo maior rebanho do mundo e $1^{\circ}$ lugar em exportação. De forma semelhante, tem-se a avicultura, onde o país é líder do ranking mundial de exportação de carne de frango, detendo cerca de $40 \%$ do comércio mundial e ocupa o $3^{\circ}$ lugar em volume de produção. Logo atrás, os suínos, onde o país ocupa a $4^{a}$ posição tanto em produção como em volume de exportação. Fica evidente, então, que o país tem tradição e ocupa um lugar de destaque na produção e exportação de proteína animal no mundo. Cabe sublinhar a participação de destaque do estado de Santa Catarina na produção de suínos e aves, sendo um dos principais produtores no Brasil.

Já no que diz respeito à exportação de pescados, seja na aquicultura continental ou na maricultura, o país não tem apresentado um desempenho condizente com seu potencial de produção aquícola. A balança comercial de pescados historicamente apresenta déficit. A produção atual, tanto da pesca quanto da aquicultura, não tem sido suficiente para suprir a demanda interna do país, incorrendo em grandes volumes de importações recorrentes.

Do ponto de vista da produção global de pescados, o grupo dos moluscos ocupa o segundo lugar no volume de produção mundial, voltada para alimentação humana e oriunda de cultivo, com 24\% de toda a produção, equivalentes a uma quantidade de 16.113.200 toneladas, conforme dados da figura 3 (FAO, 2016).

Figura 3 - Produção aquícola em 2014. (plantas aquáticas não inclusas).

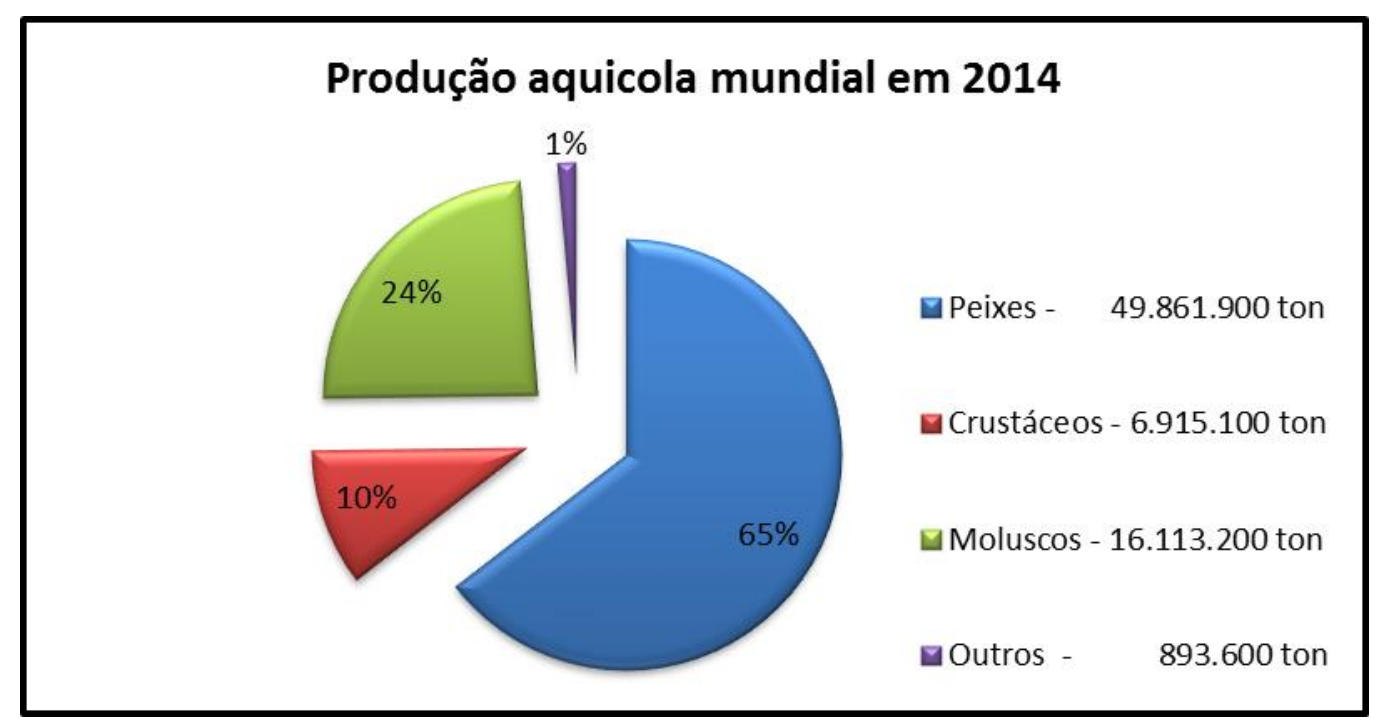


Maricultura em Santa Catarina: a cadeia produtiva gerada pelo esforço coordenado de pesquisa, extensão e desenvolvimento tecnólogico

Fonte: FAO, 2016

É indiscutível a importância dos resultados já alcançados com a maricultura em Santa Catarina, especialmente para as pessoas e empresas diretamente envolvidas na atividade de produção de bivalves. Lembrando que a malacocultura catarinense representa, cerca de 98\% de produção de bivalves no Brasil. Apesar deste desempenho de destaque nacional, quando se assume a perspectiva global, percebe-se que ainda há muito o que se conquistar.

$\mathrm{Na}$ Figura 4, consideraremos avaliar apenas o grupo de moluscos, mais especificamente voltando a atenção para a comparação entre o Brasil e o líder mundial, a China, com 83\% de toda a produção, e o líder da América Latina, o Chile, com a produção de 246.400 ton. A produção do Brasil de 22.100 toneladas é equivalente a $9 \%$ da produção do vizinho Chile e a apenas $0,14 \%$ da produção mundial.

Figura 4: Produção mundial de moluscos da China, Chile, Brasil e outros.

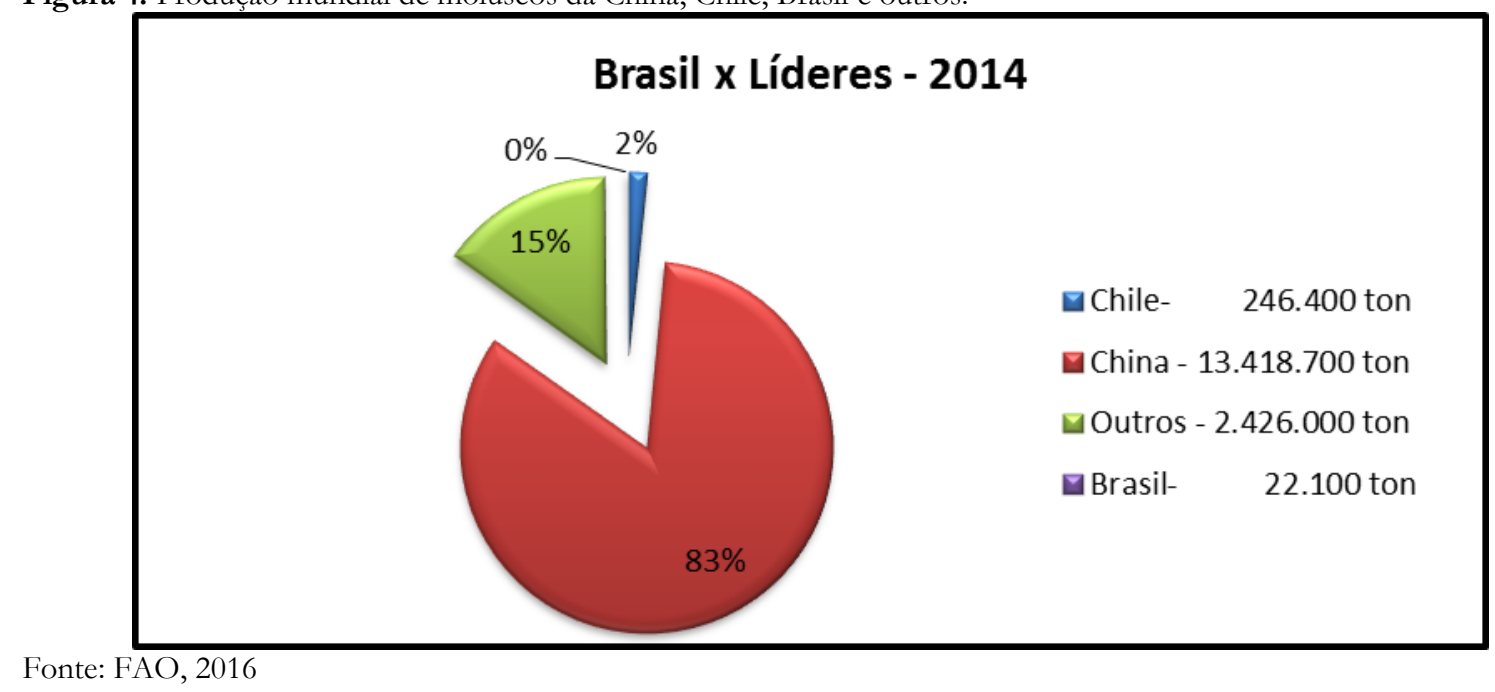

A China assume a posição de líder isolado no ranking mundial, com produção mais de quatro vezes superior ao total produzido por todo resto do mundo, e, portanto, se distancia muito da realidade nacional.

Todavia, o Chile pode ser uma referência interessante pelo histórico de crescimento. Em 1990, teve uma produção de 3.800 ton de bivalves. Com uma visão estratégica acurada, associada a muito trabalho para a abertura de alguns mercados europeus, a produção chegou a 24.100 ton em 1997. A partir deste ponto os volumes cresceram rapidamente, em 2003 já alcançava 77.700 ton, e, em 2014 alcançou 246.400 ton. É um país com cultura exportadora, com acordos de livre comércio firmados e canais de exportação abertos, embarcando seus pescados para diversos 
Maricultura em Santa Catarina: a cadeia produtiva gerada pelo esforço coordenado de pesquisa, extensão e desenvolvimento tecnólogico

países do mundo, inclusive para o Brasil. Por exemplo, no ano de 2011, o Chile exportou 58.049 ton de bivalves apenas para União Europeia, sendo concentrados nos seguintes países: Espanha 42\%, França 20,8\%, Reino Unido 5,8\% e Holanda 4,4\%. (SERNAPESCA, 2011).

À margem de uma eventual abertura de mercado para comercialização de bivalves brasileiros no exterior apresenta-se um breve diagnóstico da situação atual da malacocultura catarinense.

Quanto à análise do ambiente interno (Santa Catarina e Brasil), tem-se:

\section{PONTOS FORTES}

Cadeia de produção já estabelecida e operando continuamente a mais de 20 anos.

As atuais áreas produtivas, já licitadas, ainda estão longe do esgotamento da capacidade produtiva. Em 2014, ainda restavam 70\% de capacidade disponível (EPAGRI, 2015).

Litoral catarinense é extenso, com condições ambientais e geográficas favoráveis para ampliação de áreas produtivas.

$\checkmark \quad$ Reconhecimento da excelência e tradição do estado como produtor de moluscos de alta qualidade.

As espécies hoje produzidas apresentam excelente desempenho zootécnico no ambiente de Santa Catarina. As ostras do pacífico apresentam um crescimento muito rápido, atingem o tamanho comercial em 8 meses (MANZONI, 2001). Na França para atingir o mesmo tamanho os animais levam de 2 a 4 anos (GOSLING, 2004).

$\checkmark \quad$ Implantação de programas de controle sanitário (PNCMB), em 2012, e das regras de conduta com a utilização das melhores práticas de produção.

$\checkmark \quad$ Desenvolvimento de técnicas para maturação de reprodutores em laboratório e assentamento larval remoto.

$\checkmark \quad$ Pesquisa aplicada na área de melhoramento genético no cultivo de bivalves visando melhorar o desempenho zootécnico dos animais.

\section{PONTOS FRACOS}

Método de produção ainda artesanal, sem uso intensivo de mecanização, como resultado tem-se relativa baixa produtividade nos cultivos.

$\checkmark \quad$ Dificuldades em ampliar a demanda no mercado interno devido, entre outros fatores, às questões de ordem cultural (hábitos alimentares) e, portanto com alta resistência para mudanças. 
Maricultura em Santa Catarina: a cadeia produtiva gerada pelo esforço coordenado de pesquisa, extensão e desenvolvimento tecnólogico

$\checkmark \quad$ Pouca diversificação de produtos.

Tamanho das áreas dos atuais parques aquícolas são, em média, pequenas, de até 2 hec Sendo que para o uso de modelo mecanizado de cultivo, as áreas a partir de 10 hec são mais adequadas.

Devido a fatores favoráveis em SC (áreas abrigadas, temperatura, nutrientes e salinidade), tem-se grande produção primária, que por sua vez favorece também a propagação de organismos competidores (incrustadores) com os moluscos, tornando o manejo uma atividade frequente.

Processos produtivos pouco ergonômicos e potencialmente prejudiciais à saúde do trabalhador.

$\checkmark \quad$ A produção e oferta de formas jovens de bivalves ficam concentradas em poucos fornecedores.

$\checkmark \quad$ Baixo investimento em saneamento público.

Quanto à análise do ambiente externo (Mercado Externo), tem-se:

\section{OPORTUNIDADES}

Processo de adequação das normas nacionais de produção e controle sanitário aos requisitos já aplicados em outros grandes produtores (JACOMEL, 2014; SOUZA et al., 2014).

$\checkmark \quad$ Esgotamento das áreas disponíveis para cultivo de moluscos em outros países considerados grandes produtores e consumidores desses produtos, como é o caso de França e Espanha (VARADI et al., 2001).

$\checkmark$ Pacotes tecnológicos, voltados para uso intensivo de mecanização, já desenvolvidos e em pleno funcionamento em outros países.

$\checkmark \quad$ Padronização das Melhores Práticas de Produção e disseminação destas em nível global.

Mercado mundial de moluscos movimenta cerca US\$19 bilhões ao ano (FAO,2016).

$>$ AMEAÇAS

$\checkmark \quad$ Novas doenças e patógenos que tem causado mortalidades em massa no cultivo de bivalves em fazendas na Europa (RENAUT, 1996 ; SABRY et al., 2009). 
Maricultura em Santa Catarina: a cadeia produtiva gerada pelo esforço coordenado de pesquisa, extensão e desenvolvimento tecnólogico

$\checkmark \quad$ Problemas de ordem maior, com origem na degradação ambiental mundo afora, podem prejudicar seriamente o cultivo de moluscos, como é o caso da possibilidade de acidificação dos oceanos (TURLEY \& GATTUSO, 2012).

Entrada e crescimento das importações de bivalves chilenos dentro do mercado nacional.

$\checkmark \quad$ Aumento da contaminação dos oceanos por poluição doméstica e industrial.

$\checkmark$ Com aumento da carga orgânica que chega aos oceanos, através da poluição, crescem as ocorrências das "marés vermelhas".

$\checkmark \quad$ Surgimento e disseminação de novos parasitas de bivalves.

Com base nas informações apresentadas nesta análise é possível imaginar que a próxima década possa ser extremamente promissora para a Cadeia de Produção de Moluscos em Santa Catarina, se considerarmos o potencial de abertura de mercado. Certamente, a exportação dos produtos aqui gerados pode significar uma verdadeira revolução econômica e social. Desta forma, o resultado poderá ser de um crescimento radical dos volumes de produção, até mesmo superior ao crescimento já vivenciado na década de 90, proporcionando incrementos vultosos na movimentação financeira e na geração de empregos.

Contudo, é sabido que, até estar apto à exportação, um longo caminho precisa ser percorrido. Este caminho têm sido e ainda será de constante evolução, às despensas de muito trabalho. O elevado grau de competitividade dos países que participam do comércio internacional de moluscos exige mudanças importantes na cadeia produtiva, tais como: planejamento estratégico orientado para exportação, criação de maior inteligência comercial, adequação de produtos aos mercados alvos, diversificação de produtos, aumento da capacidade de produção tanto em tamanho quanto em aprimoramento tecnológico, melhoria de processos, ampliação da mecanização, sistematização da gestão, definição de estratégias para divulgação e promoção comercial, estabelecimento de acordos de livre comércio, aprimoramento da estrutura logística e, por fim, a comercialização.

À vista do desempenho exemplar do Chile, através de um trabalho organizado e coordenado entre entes públicos, produtores de bivalves e das instituições de pesquisa e extensão, acredita-se que Santa Catarina tenha todos os elementos necessários para iniciar uma nova era no estado, com a modernização dos cultivos e o foco de produção voltada para atender, além do mercado interno, o imenso mercado mundial de consumo de moluscos bivalves. 
Maricultura em Santa Catarina: a cadeia produtiva gerada pelo esforço coordenado de pesquisa, extensão e desenvolvimento tecnólogico

\section{AGRADECIMENTOS}

Pela ajuda na elaboração deste trabalho agradecemos a toda equipe que trabalha no Laboratório de Moluscos Marinhos da UFSC e que viabiliza, até os dias atuais, a atividade da malacocultura em SC. Em especial: ao Prof. Claudio Melo, ao Prof. Marcos Caivano, a Francisco da Silva, a Jaqueline Araújo, a Claúdio Blacher e a Carlos Henrique Aráujo.

\section{REFERÊNCIAS}

EPAGRI 2015. Síntese Informativa da Maricultura 2014. Florianópolis: EPAGRI. 2015.

EPAGRI 2016. Síntese Informativa da Maricultura 2015. Florianópolis: EPAGRI. 2016.

FAO 2016. The State of World Fisheries and Aquaculture 2016. Contributing to food security e nutrition for all. Rome: Food and agriculture organization, 2016. 200p.

GOSLING, E. 2004. Bivalves Molluscs: biology, ecology and culture. Oxford: fishing new books. Blackwell publishing company. 443p.

HELM, M.M.; BOURNE, N.; Lovatelli, A. Hatchery culture of bivalves. A practical manual. FAO Fisheries Technical Paper. No. 471. Rome, FAO. 2004. 177p.

HINCKEL, J. P.. Carbon sequestration potential of shellfish. Technical report, School of Natural and Built Environs, LMES, University of South Australia. Seminars in Sustainability-UniSA, 2008.

HUNER, J. V. \& BROWN, E. E. Crustacean and mollusk aquaculture in the United States. AVI publishing company, INC. Westport, Connecticut. 476: 235-273.

IBGE 2015. Produção da Pecuária Municipal. Brasília: Instituto Brasileiro de Geografia e Estatística, 2015.36p.

JACOMEL, B. Produção sustentável e controlada de ostras: ações em Santa Catarina rumo aos padrões internacionais de comercialização. Dissertação- Departamento de Engenharia de Produção e Sistemas, Universidade Federal de Santa Catarina, Florianópolis. 2014.

MANZONI, G. 2001. Ostras aspectos bioecológicos e técnicas de cultivo. Univali, CTTmar: CGMA. 30p.

POLI, C. R. Cultivo de ostras do pacífico (C. gigas). In: Aquicultura. Experiências Brasileiras. Florianópolis: Multitarefa, p. 251-266. 2004.

QUAYLE, D. B. \& NEWKIRK, G. F. Farming bivalve mollusks: Methods for Study and Development. In: Sandifer, P. A. (ed). Advances in Word Aquaculture. 1989, v.1, 273p. 
Maricultura em Santa Catarina: a cadeia produtiva gerada pelo esforço coordenado de pesquisa, extensão e desenvolvimento tecnólogico

RENAULT, T. Appearance and spread of diseases among bivalve molluscs in the northern hemisphere in relation to international trade Rev. sci. tech. Off. int. Epiz., 1996, 15 (2), 551-561

SERNAPESCA. Relatório de exportação. Exportacion Mytilus chilensis 2011. Acessado em www.senarpesca.cl em 27/09/2016.

SOUZA, R. V. de; RUPP, G. S.;CAMPOS, C. J. A. de; LEE, R. Moluscos bivalves: medidas de controle microbiológico para atender às exigências da União Européia. Florianópolis: Epagri, 2014, 48p.

SABRY, R.C., R.D. ROSA, A.R.M. MAGALHÃES, M.A. BARRACCO, T.C.V. GESTEIRA and P.M. da Silva. 2009. First report of Perkinsus sp. infecting mangrove oysters Crassostrea rbizophorae from the Brazilian coast. Diseases of Aquatic Organisms. 2009, 88: 13-23.

TURLEY, C. \& GATTUSO, J. P. Future biological and ecosystem impacts of ocean acidification and their socioeconomic policy implications. Current Opinion in Environmental Sustainability. 2012, 4:1-9.

VARADI, L., SZUCS, I., PEKAR, F., BLOKHIN, S. \& CSAVAS , I. Aquaculture development trends in Europe. In: Technical Proceedings of the Conference on Aquaculture in the Third Millennium, Bangkok, Thailand, 2001.

WOLF, J. G. \& BEAUMONT, A. 2011. Shellfish Sequestration: The Augmented Cultivation of Molluscs, and the Preservation of their Shells, as a Means of Sequestering Carbon Dioxide. Acessado em 04/10/16 - www.mng.org.uk/gh/private/ssr7a.pdf 